\title{
Erratum: XXXIII Congresso Nazionale EDTNA/ERCA - Bari, 14-16 Maggio 2015
}

Informiamo i nostri lettori che nel seguente abstract:

Giornale di Tecniche Nefrologiche e Dialitiche 2015 Apr-Giu;27(2):99-114. DOI: 10.5301/GTND.2015.14648.

Abstract P 044

Il counseling infermieristico nella nefrologia diabetica.

Stipa R., Di Massimo A.R.

U.O.C. Nefrologia e Dialisi, Ospedale Riuniti di Anzio e Nettuno, Anzio - Nettuno (RM)

Gli Autori e le affiliazioni corretti sono i seguenti:

di Massimo A.R. ${ }^{1}$, Stipa D. ${ }^{2}$

${ }^{1}$ UOC Nefrologia e Dialisi, O.O.R.R. Anzio e Nettuno, Anzio - Nettuno (RM)

${ }^{2}$ Infermiera Professionista, Nettuno (Roma) 